\title{
Case Report \\ Community-acquired methicillin-resistant Staphylococcus aureus pyomyositis in an intravenous drug user
}

\author{
Correspondence \\ A. Fowler \\ afowler@doctors.org.uk
}

Received 1 August 2005

Accepted 5 September 2005

\author{
A. Fowler and A. Mackay \\ Department of Microbiology, Queen Elizabeth Hospital, Woolwich, London SE18 4QH, UK
}

\begin{abstract}
Pyomyositis is a disease of abscess formation deep within large striated muscles. Outside of the tropics it is a rare disease which occurs mainly in certain patient populations such as the immunosuppressed or intravenous drug users (IDUs). A case of community-acquired methicillin-resistant Staphylococcus aureus (CA-MRSA) pyomyositis in an IDU is described. The incidence of both CA-MRSA and pyomyositis is currently increasing. To the authors' knowledge this is the first reported case of CA-MRSA pyomyositis in the UK. Cases of CA-MRSA pyomyositis are likely to increase and it may be necessary to empirically treat certain patients with glycopeptides.
\end{abstract}

\section{Introduction}

Pyomyositis was first described in the tropics and is often called tropical pyomyositis. It is an abscess-forming infection of skeletal muscle (Ebright \& Pieper, 2002). It is a rare disease and was only recognized as a temperate condition as recently as 1971 (Levin et al., 1971). The disease is characterized by cramping pains, followed by oedema, worsening discomfort and mild fever. The muscle may be indurated with oedema and tenderness increasing as the infection progresses. Obvious fluctuance develops in about half the patients. As the infection progresses the patient may become increasingly systemically unwell with septicaemia. The abscesses may develop via spread from a haematogenous route or from contiguous soft tissue or bone. The most common sites of abscess formation are the quadriceps, gluteus, shoulder and upper arm muscles. Multiple foci occur in about $40 \%$ of patients. It is caused in $75 \%$ of cases by Staphylococcus aureus (Bickels et al., 2002). A review of the literature, using searches by Medline and EMBASE, demonstrated that MRSA is an infrequent cause of the infection with the first ever case report in 1996 (Kong et al., 1996).

\section{Case report}

A 21-year-old Caucasian woman presented with a 2-week history of pain and swelling in the right thigh. There was no history of trauma or immobilization. She gave a history of intravenous drug use, sometimes injecting into her groin. She had no other relevant past medical history and no previous hospital admissions. On examination she was

Abbreviations: CA-MRSA, community-acquired methicillin-resistant Staphylococcus aureus; DVT, deep vein thrombosis; ICU, intensive care unit; IDU, intravenous drug user. apyrexial with a tachycardia of 100 b.p.m., a blood pressure of 99/60 mmHg and her thigh was swollen, tender and warm. Laboratory results showed: haemoglobin $11.7 \mathrm{~g} \mathrm{dl}^{-1}$; white cell count $24 \cdot 3 \times 10^{9} \mathrm{l}^{-1}$; neutrophils $22.9 \times 10^{9} \mathrm{l}^{-1}$; platelets $682 \times 10^{9} \mathrm{l}^{-1}$; D-dimer positive; urea $20 \cdot 0 \mathrm{mmol} \mathrm{l}^{-1}$; creatinine $112 \mu \mathrm{mol} \mathrm{l}^{-1}$. A clinical diagnosis of deep vein thrombosis (DVT) with superadded cellulitis was made. She was admitted for intravenous hydration, anticoagulant therapy and oral antimicrobial treatment with flucloxacillin (500 mg q.d.s.). She was subsequently switched to intravenous flucloxacillin ( $1 \mathrm{~g}$ q.d.s.), and benzylpenicillin $(1 \cdot 2 \mathrm{~g}$ q.d.s.) was added to the antibiotic treatment regimen.

The patient then deteriorated with worsening pain and swelling of the thigh and groin. She became pyrexial $\left(39^{\circ} \mathrm{C}\right)$, increasingly tachycardic (128 b.p.m.) and hypotensive (70/ $40 \mathrm{mmHg}$ ). The white cell count had risen to $46 \times 10^{9} \mathrm{l}^{-1}$ (neutrophils $40 \times 10^{9} \mathrm{l}^{-1}$ ). Imaging with ultrasound ruled out a DVT but computed tomography (CT) revealed grossly swollen iliopsoas and upper thigh muscles and possible muscle necrosis. Magnetic resonance imaging (MRI) showed grossly swollen, high signal upper thigh muscles with abscess formation. Flucloxacillin was subsequently switched to meropenem ( $1 \mathrm{~g}$ b.d.) and the dose of benzylpenicillin was increased $(2.4$ g q.d.s.). A single dose of gentamicin (120 mg) was also administered.

Forty-eight hours after admission the patient underwent extensive surgical debridement of the right quadriceps muscles. The patient was subsequently admitted to the intensive care unit (ICU). Gram-positive cocci and numerous white cells were seen on microscopy of the necrotic material excised at operation. Sodium fusidate was added to the antibiotic regimen $(500 \mathrm{mg}$ t.d.s.). The necrotic material 
was processed by inoculating the sample onto several agar plates including horse blood agar (HBA; CM0271, Oxoid); MacConkey agar (CM0007; Oxoid); chocolate agar incubated in $5 \% \mathrm{CO}_{2}$ at $37^{\circ} \mathrm{C}$; $\mathrm{HBA}$ with colistin and nalidixic acid incubated in air; Columbia blood agar (CBA; CM0331, Oxoid) with and without neomycin aerobically and anaerobically for 2 and 5 days, respectively. Blood culture specimens were processed using the BACTEC 9240 system (Becton Dickinson Microbiology). The cultures were sterile after 5 days of routine incubation in this system.

The culture from necrotic tissue yielded a moderate growth of Gram-positive cocci. MRSA and Streptococcus pyogenes were identified using slide coagulase and tube coagulase systems and the API 20 Strep (bioMérieux) method of identification. Antibiotic sensitivity was tested on Iso-Sensitest agar (ISA; CM0471, Oxoid) according to the British Society of Antimicrobial Chemotherapy (BSAC) standardized disc susceptibility testing method (Andrews, 2001). The Streptococcus pyogenes was fully sensitive, whilst the MRSA strain was resistant to erythromycin and ciprofloxacin, compatible with our local strain of epidemic MRSA (EMRSA), which is EMSRA-15.

Antibiotic therapy was changed to include vancomycin ( $1 \mathrm{~g}$ b.d.), on which the patient finally began to improve. Of note, she was HIV-negative. Further debridement and exploration of the wound was required on six occasions. Ten days after admission she was discharged from the ICU and the antibiotics were stopped. The patient was discharged from hospital 4 months after admission.

\section{Discussion}

The first case report of MRSA pyomyositis in the literature was from Singapore in 1996 (Kong et al., 1996). Since then there have only been 12 further cases reported worldwide (Hayashi et al., 2003; Millar et al., 2000; Liew et al., 1998; Milochau et al., 1997; Endoh et al., 2000; Fan et al., 2002; Vassilopoulos et al., 1997; Ruiz et al., 2005) and in 9 of these the MRSA was acquired from the community (Kong et al., 1996; Liew et al., 1998; Milochau et al., 1997; Fan et al., 2002; Vassilopoulos et al., 1997; Ruiz et al., 2005). Most recently, a case series of four patients was reported in the USA (Ruiz et al., 2005). The MRSA was community-acquired, leading the authors to conclude that CA-MRSA is emerging as an important cause of pyomyositis. To our knowledge this is the first case of CA-MRSA pyomyositis to be reported in the UK.

Despite being a rare disease, the incidence of pyomyositis is increasing in Western countries of the world (Hsueh et al., 1996; Skoutelis et al., 1993). There is an association between intravenous drug use and pyomyositis (Ebright \& Pieper, 2002). This is thought to be due to impaired cellular and humoral immunity and abnormal skin bacterial colonization along with the repeated introduction of nonsterile fluids (Hsueh et al., 1996). Other at-risk populations include patients with HIV, diabetes mellitus and malignancy. A rising number of such patients will result in a greater number of cases of pyomyositis in the future (Harbarth \& Lew, 1997).

Infections due to MRSA are increasing within the community (McCarthy, 2003). There is mounting evidence that MRSA is being isolated in patients who have not been hospitalized and that it may also arise de novo (Salmenlinna et al., 2002), with reports of infection with MRSA in adults and children without known risk factors (Charlebois et al., 2002). Although healthy persons are at low risk of colonization, IDUs are thought to be at risk. An outbreak of a single clone of MRSA has been described in Zurich among IDUs (Fleisch et al., 2001) and a large prevalence based study in San Francisco showed that intravenous drug use is a significant risk factor for community colonization of MRSA (Charlebois et al., 2002). In the UK two centres have recently reported cases of groin abscesses and endocarditis in IDUs caused by MRSA from the community (Anon, 2003). We predict, therefore, that there will be an increase in the incidence of CA-MRSA pyomyositis, particularly amongst IDUs.

This potential rise is of concern because pyomyositis is difficult to diagnose and prompt treatment is required to prevent complications. The difficulties and delays in diagnosis arise for several reasons (Harbarth \& Lew, 1997). Firstly, the presentation is often vague, particularly if the affected muscle is deeply sited and local signs are not apparent (Bickels et al., 2002); secondly, there is a broad spectrum of clinical presentation; and thirdly, as the infection is so rarely seen in temperate climates, it is less likely to be considered. Other diagnoses (e.g. DVT) will be considered first (Valeriano-Marcet et al., 2003), particularly in an IDU, where DVT is common. Correct therapy may therefore be significantly delayed, a delay which is exaggerated in patients with CA-MRSA pyomyositis being treated with conventional antibiotics. A delay in diagnosis can lead to joint destruction, lung and brain abscesses as well as sepsis and occasionally death (Bickels et al., 2002). In IDUs who receive appropriate treatment, mortality from pyomyositis is quoted as less than $10 \%$ (Ebright \& Pieper, 2002).

In summary, we report the first case of CA-MRSA pyomyositis in the UK and suggest that the incidence of this disease may increase in the future both here and abroad. We postulate that, in the UK and other countries with an increasing burden of CA-MRSA, it may be necessary to consider including a glycopeptide in the initial treatment of pyomyositis, until cultures grow sensitive organisms, in high-risk patient populations such as IDUs even if the patient is admitted from the community. This option must also be considered if patients are failing to respond to conventional treatment. We acknowledge that this attitude may place even greater selection pressure on the spread of vancomycin-resistant strains of MRSA. We feel, however, that clinicians should carefully consider the use of a glycopeptide antibiotic in the initial treatment of such cases 
because there are such devastating outcomes in patients in whom correct therapy is delayed.

\section{References}

Andrews, J. M. (2001). BSAC standardized disc susceptibility testing method. BSAC Working Party on Susceptibility Testing. J Antimicrob Chemother 48, 43-57.

Anonymous (2003). Methicillin-resistant Staphylococcus aureus (MRSA) in injecting drug users. CDR Weekly 13 (Suppl. 27), 3.

Bickels, J., Ben-Sira, L., Kessler, A. \& Wientroub, S. (2002). Primary pyomyositis. J Bone Joint Surg Am 84-A, 2277-2286.

Charlebois, E. D., Bangsberg, D. R., Moss, N. J., Moore, M. R., Moss, A. R., Chambers, H. F. \& Perdreau-Remington, F. (2002). Populationbased community prevalence of methicillin-resistant Staphylococcus aureus in the urban poor of San Francisco. Clin Infect Dis 34, 425-433.

Ebright, J. R. \& Pieper, B. (2002). Skin and soft tissue infections in injection drug users. Infect Dis Clin North Am 16, 697-712.

Endoh, K., Kawai, N., Itoh, K. \& 8 other authors (2000). Improvement of subcutaneous and intramuscular MRSA abscesses, which developed after treatment of acute myelocytic leukaemia, by treatment with minocycline and arbekacin. Jpn J Chemother 48, 747-750.

Fan, H. C., Lo, W. T., Chu, M. L. \& Wang, C. C. (2002). Clinical characteristics of Staphylococcal pyomyositis. J Microbiol Immunol Infect 35, 121-124.

Fleisch, F., Zbinden, R., Vanoli, C. \& Ruef, C. (2001). Epidemic spread of a single clone of methicillin-resistant Staphylococcus aureus among injection drug users in Zurich, Switzerland. Clin Infect Dis 32, 581-586.

Harbarth, S. J. \& Lew, D. P. (1997). Pyomyositis as a nontropical disease. Curr Clin Top Infect Dis 17, 37-50.

Hayashi, T., Nozaki, M., Nonaka, Y., Ohashi, K., Sakamaki, H. \& Nomura, T. (2003). Pyomyositis as a focus of infection in haematological disorders: a report of three cases. Int J Hematol 77, 171-174.

Hsueh, P. R., Hsiue, T. R. \& Hsieh, W. C. (1996). Pyomyositis in intravenous drug abusers: report of a unique case and review of the literature. Clin Infect Dis 22, 858-860.

Kong, N. C., Asmah, J., Lim, V. K., Ong, P. H. \& Adam, P. A. (1996). Pyomyositis revisited. Ann Acad Med Singapore 25, 609-611.

Levin, M. J., Gardner, P. \& Waldvogel, F. A. (1971). An unusual infection due to staphylococcus aureus. N Engl J Med 284, 196-198.

Liew, K. L., Choong, C. S., Liu, P. N., Tsai, D. H., Chen, L. H. \& Yang, W. C. (1998). Pyomyositis in childhood: a case report. Chung-Hua I Hsueh Tsa Chih - Chin Med J 61, 488-491.

McCarthy, M. (2003). Resistant bacteria spread through US communities. Lancet 362, 1554.

Millar, C., Page, T., Paterson, P. \& Taylor, C. P. (2000). MRSA pyomyositis complicating sickle cell anaemia. Clin Lab Haematol 23, 329-332.

Milochau, P., Talarmin, B., Le Ru, Y., Allard, G. \& Plantin, P. (1997). [Methi-resistant Staphylococcus aureus myositis]. Ann Dermatol Venereol 124, 612-614.

Ruiz, M. E., Yohannes, S. \& Wladyka, C. G. (2005). Pyomyositis caused by methicillin-resistant Staphylococcus aureus. N Engl J Med 352, 1488-1489.

Salmenlinna, S., Lyytikäinen, O. \& Vuopio-Varkila, J. (2002). Community acquired methicillin-resistant Staphylococcus aureus, Finland. Emerg Infect Dis 8, 602-607.

Skoutelis, A., Andonopoulos, A., Panagiotopoulos, E. \& Bassaris, H. (1993). Non-tropical pyomyositis in adults: report of four cases and literature review. Eur J Clin Microbiol Infect Dis 12, 769-772.

Valeriano-Marcet, J., Carter, J. D. \& Vasey, F. B. (2003). Soft tissue disease. Rheum Dis Clin North Am 29, 77-88.

Vassilopoulos, D., Chalasani, P., Jurado, R. L., Workowski, K. \& Agudelo, C. A. (1997). Musculoskeletal infections in patients with human immunodeficiency virus infection. Medicine 76, 284-294. 